\title{
Comunicação de Dados em Redes de Distribuição de Energia Elétrica de Baixa Tensão
}

\author{
Richard Brandão Nogueira Vital \\ Comissão Nacional de Energia Nuclear \\ richardvital@gmail.com \\ Tatiane Melo Vital \\ Instituto Nacional de Telecomunicações \\ tati.mv7@gmail.com
}

\begin{abstract}
Resumo: Este artigo descreve o mecanismo de troca de informações com uso de redes brasileiras de distribuição de energia elétrica de baixa tensão, e traça a linha evolutiva do tema até atingir o desenvolvimento tecnológico atual. Dessa forma, são abordados temas importantes sobre o assunto como aplicações comerciais, faixa de operação, descrição dos dispositivos de rede que possibilitam a troca de dados, comportamento do canal de comunicação e das fontes ruidosas.
\end{abstract}

Palavras-chave: Tecnologia PLC. Modem. Canal. Ruído.

\section{Low-Voltage Power Line Communication}

\begin{abstract}
This paper describes a communication mechanism using Brazilian low voltage electrical grid, showing the evolution until the actual state of the art. Thus, important issues about the technology were showed like: commercial applications, available band, communication devices, channel and noise characterization.
\end{abstract}

Keywords: Power Line Communication. Modem. Channel. Noise.

\section{Introdução}

Devido ao crescimento da demanda por eficientes e competitivos meios de troca de informações, diversas tecnologias têm sido pesquisadas e desenvolvidas. Nesse cenário, a transmissão de dados em que se utiliza a infraestrutura de distribuição de energia elétrica vem ganhando destaque, uma vez que apresenta características importantes como baixo custo de implantação, acessibilidade de grande parte dos potenciais consumidores e facilidade de implantação (Götz, Rapp e Dostert, 2004).

A Tecnologia PLC começou a ser utilizada na década de 30 por empresas concessionárias de energia elétrica. A utilização da tecnologia em redes de alta-tensão vislumbrava serviços de monitoração de desempenho e segurança das linhas, serviço que ficou conhecido como 
OPLAT (Onda Portadora em Linha de Alta-Tensão). Atualmente, a utilização de linhas de transmissão de energia elétrica desperta interesse nas aplicações de comunicação de dados a alta velocidade em redes de baixa tensão, também conhecida como BPL (Broadband over Powerline). A solução BPL já é comercializada e provê taxas de transferência de dados superiores a $200 \mathrm{Mbps}$.

A utilização das redes de energia elétrica como meio de transmissão vem sendo estudada e testada em diversas partes do mundo, principalmente nos Estados Unidos e Europa, com destaque para a HomePlug (HomePlug Powerline Alliance, 2006) e o projeto OPERA (Open PLC European Research Alliance, 2005). No Brasil existem projetos relacionados às concessionárias de energia elétrica (COPEL, LIGHT, CEMIG, ELETROPAULO, CEMAR e CELG) e projetos relacionados à inclusão digital nas seguintes cidades: Barreirinhas-MA, Restinga-RS, Candiota-RS e Pedras-PE (APTEL, 2003).

\section{Tecnologia PLC}

A Tecnologia PLC consiste na transmissão de dados, com utilização da faixa de frequência compreendida entre 1 e $30 \mathrm{MHz}$, devido às características do meio de transmissão que introduz alta degradação fora dessa faixa. No Brasil, a Resolução 527/09, da ANATEL, define duas faixas para a utilização do serviço: a de 1,7 a $30 \mathrm{MHz}$, destinada a aplicações com distância de até $30 \mathrm{~m}$, e a de 30 a $50 \mathrm{MHz}$ para serviços de distâncias curtas, tipicamente 3 metros.

A transmissão de dados em banda larga, objeto de estudo desse trabalho, vislumbra a implementação de diversos serviços, dentre os quais se destacam: acesso em banda larga à internet; vídeo sob demanda; tele-medicina; aplicações de TVs e rádios digitais; serviços de monitoração e segurança; automação residencial, comercial e industrial; monitoração de processos produtivos (Götz, Rapp e Dostert, 2004). A figura 1 mostra um exemplo de comunicação PLC em redes domésticas de baixa tensão.

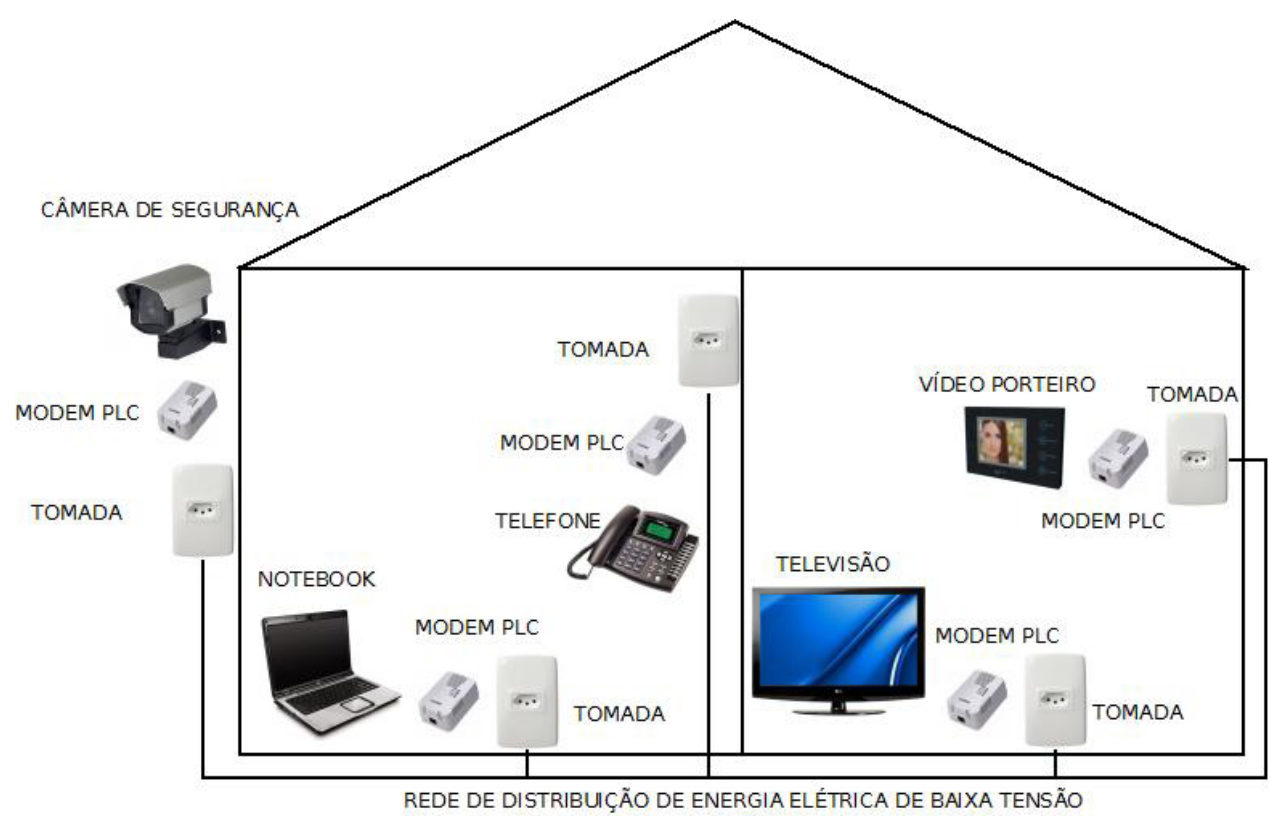

Figura 1. Exemplo de uma Rede PLC doméstica 
O dispositivo básico capaz de prover a comunicação entre diversos sistemas de comunicações é o modem PLC, que, conectado a uma tomada, possibilita a transmissão e recepção de dados oriundos de vários dispositivos com diferentes padrões e interfaces. Dependendo das características e dimensões das redes podem ser necessários outros aparelhamentos como o equipamento-mestre (TE - Transformer Equipment), responsável pela conexão da rede local e backbone de acesso à internet e, ainda, um repetidor de baixa tensão (IE - Intermediate Equipment) que desvia os sinais PLC de um medidor de consumo que pode imprimir degradação ao sinal.

Basicamente, um modem PLC é composto por um circuito de acoplamento (indutivo, capacitivo, resistivo ou por irradiação de campo eletromagnético), fonte de corrente contínua, filtros (passa-baixa ou passa-faixas), processador, conversor de protocolo (USB ou ethernet), moduladores e demoduladores digitais (M-FSK, M-PSK, M-QAM, OFDM ou espalhamento espectral). A figura 2 ilustra um diagrama em blocos simplificado de um modem PLC.

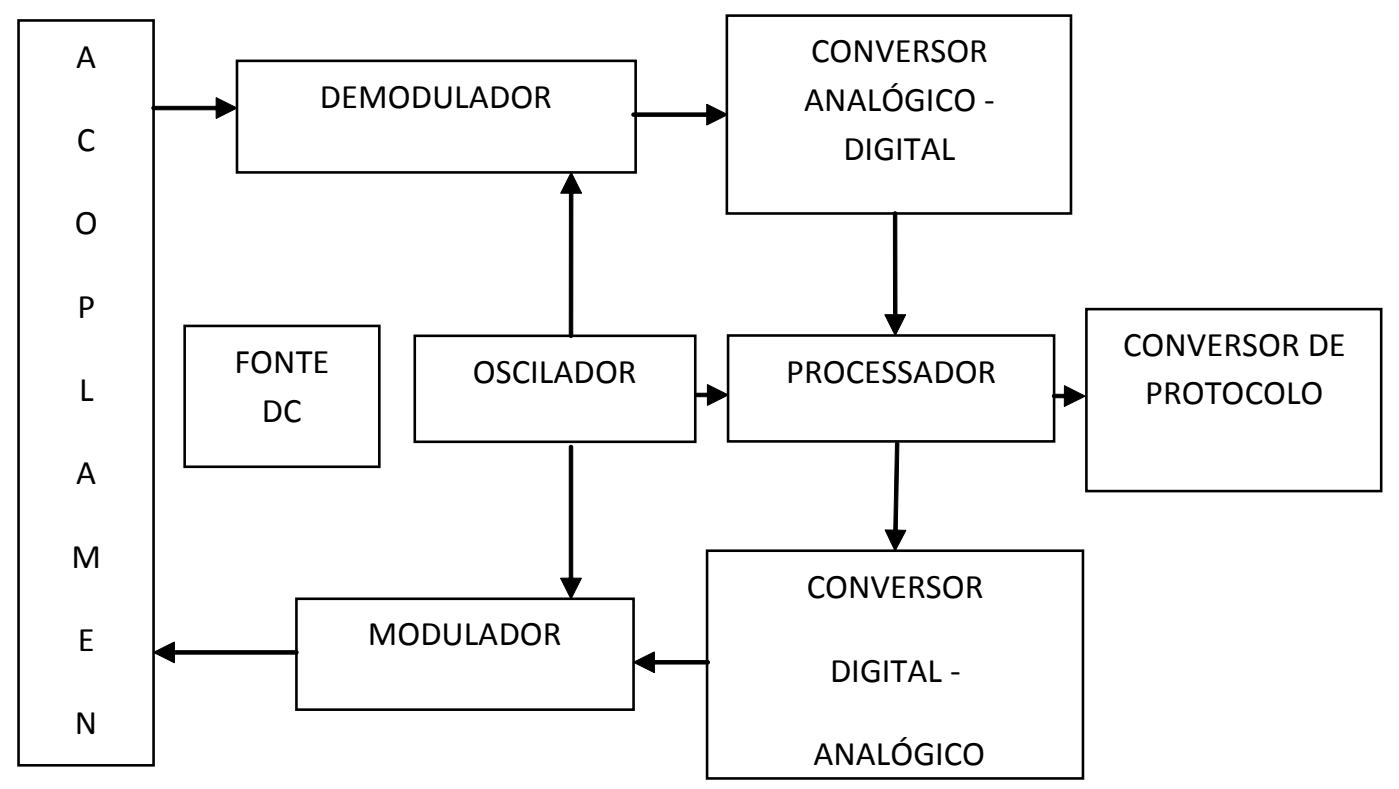

Figura 2. Diagrama em blocos simplificado de um modem PLC

Uma vez que as características do canal de comunicação estão diretamente relacionadas às condições da rede (perdas em cabeamento, emendas, tomadas, transformadores e medidores de consumo), o principal desafio dos projetistas é identificar soluções que apresentem maior robustez às características do canal e ruídos interferentes.

\section{Características do canal PLC}

Para o aperfeiçoamento ou desenvolvimento de novas demandas tecnológicas, o conhecimento das características do canal de comunicação se torna cada vez mais importante, uma vez que pode direcionar a escolha dos processos de modulação, codificação e processamento de sinais mais adequados. O canal PLC apresenta complexas 
características como o desvanecimento seletivo em frequência e a existência de múltiplos percursos de propagação que estão diretamente relacionados aos pontos de reflexão do sinal nas terminações do cabeamento elétrico (Philipps, 1999). A figura 3 mostra a interação dos agentes interferentes durante a comunicação na infraestrutura de distribuição de energia elétrica.

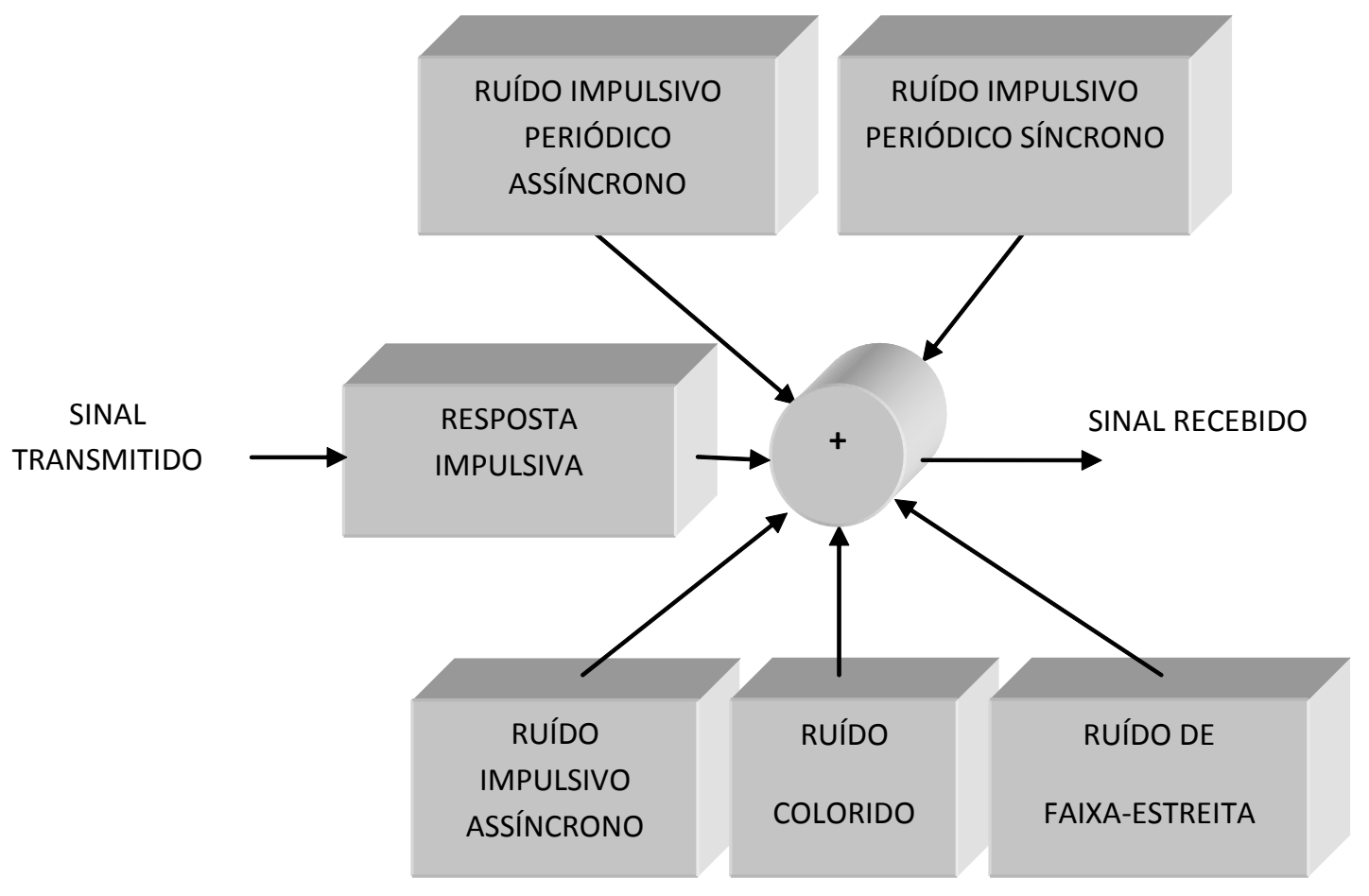

Figura 3. Representação de um canal PLC

O modelo apresentado demonstra uma possível subdivisão da caracterização do canal em dois temas: o primeiro, diz respeito à influência da resposta ao impulso do canal, o outro se relaciona às características das fontes ruidosas presentes no ambiente em estudo.

\section{Características do Canal}

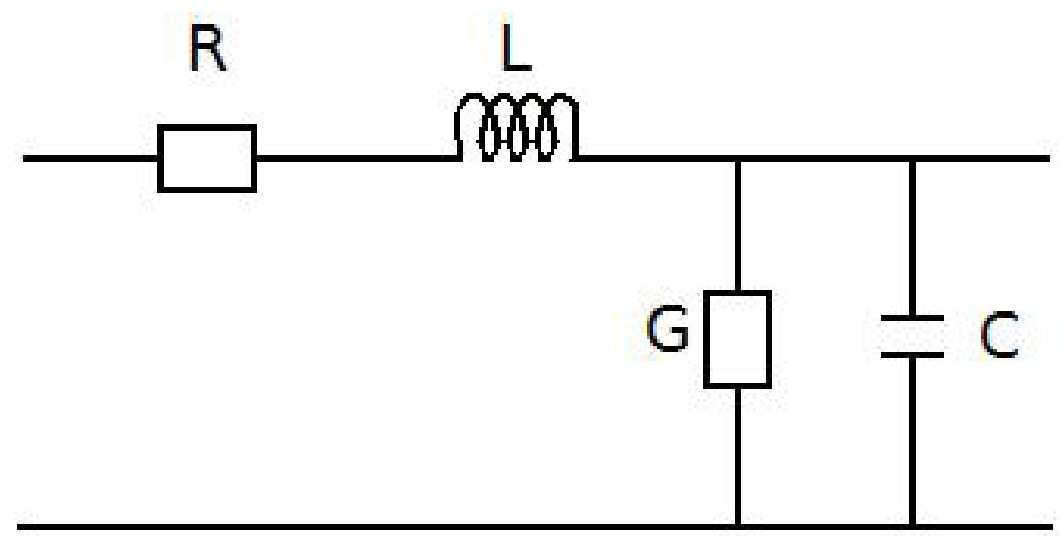

Figura 4. Modelo simplificado de uma linha de transmissão 
A linha de transmissão é modelada por uma constate de propagação complexa definida na Equação 1

$$
\gamma=\sqrt{(R+j \times \omega \times L)+(G+j \times \omega \times C)}=\alpha+j \times \beta
$$

donde $\omega$ é a frequência angular da onda eletromagnética, $\alpha$ representa a constante de atenuação e $\beta$ a constante de fase. Nesse modelo, a constante de atenuação se relaciona à degradação da onda devido às características do condutor e do isolamento, e à constante de fase que possui relação com a reatância complexa do cabeamento.

A partir das propriedades físicas da rede, defini-se que a resposta impulsiva do canal PLC é um somatório das contribuições de cada uma das $N$ componentes de múltiplo percurso $\left(g_{i}\right)$ correspondente a um determinado atraso $\left(\tau_{i}\right)$, conforme descrito na Equação 1 (Zimmermann e Dostert, 2002).

$$
h(t)=\sum_{i=1}^{N} g_{i} \times \delta\left(t-\tau_{i}\right)
$$

onde $\delta(\mathrm{t})$ é a função impulso.

Ao desenvolver a Equação 2 e adotar as considerações de Zimmermann e Dostert (2002) se obtém um modelo no domínio da frequência, definido pela Equação 3.

$$
H(f)=\sum_{i=1}^{N}\left|g_{i}\right| \times e^{\left(a_{0}+a_{1} \times f^{k}\right) \times d_{i}} \times e^{-j \times 2 \times \pi \times f \times \frac{i l}{v_{p}}}
$$

onde $f$ é a frequência do sinal; $d_{i}$ é a distância percorrida pelo sinal para o i-ésimo percurso; $v_{p}$ é a velocidade de propagação da onda eletromagnética no canal; $a_{0}$ é o nível mínimo de atenuação; $a_{l}$ é a taxa de crescimento da atenuação relacionado à freqüência e $k$ é um valor empírico no intervalo de 0,5 a 1 .

As figuras 5 e 6 mostram, respectivamente, o comportamento de magnitude e fase da reposta em frequência de um canal formado por duas tomadas distanciadas por $16 \mathrm{~m}$ de cabeamento em um laboratório de uma faculdade brasileira, em que se utilizaram os recursos e procedimentos demonstrados por Vital (2010). 


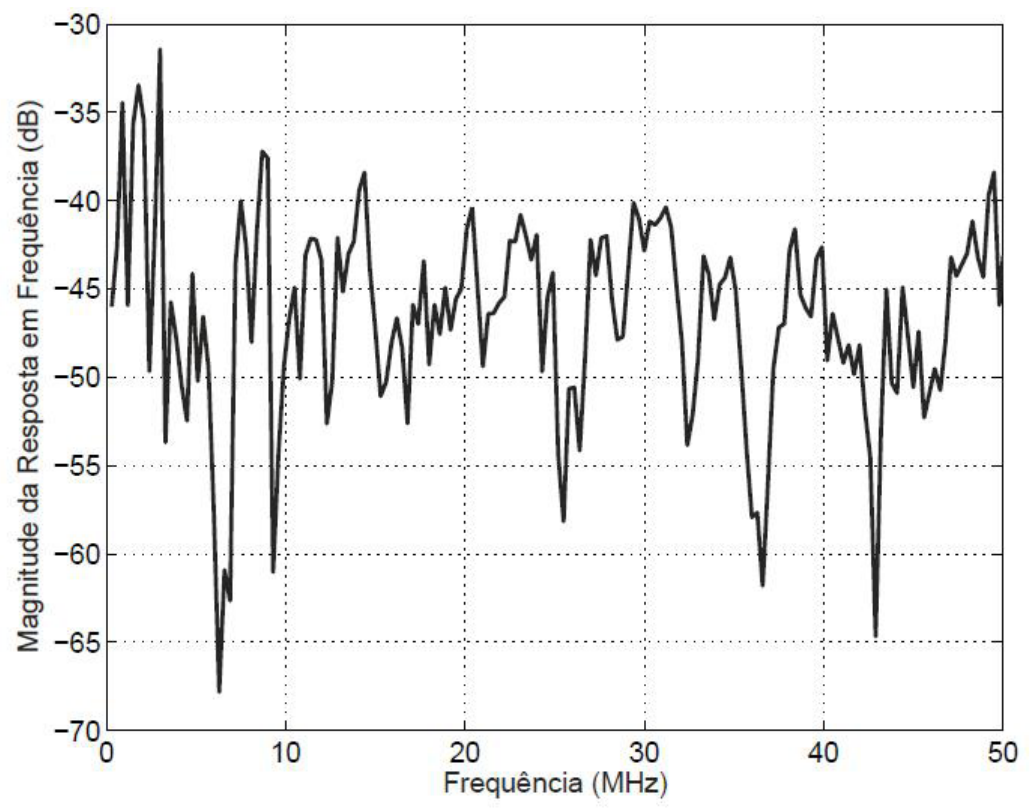

Figura 5. Magnitude da Resposta em frequência medida em um canal PLC.

Ao analisar a magnitude da resposta em frequência apresentada se observa a seletividade em frequência do canal PLC, com vales mais profundos próximos a 5 e $40 \mathrm{MHz}$. Vital (2010) apresenta um conjunto de respostas em frequência que relacionam à seletividade do canal ao comprimento do cabeamento.

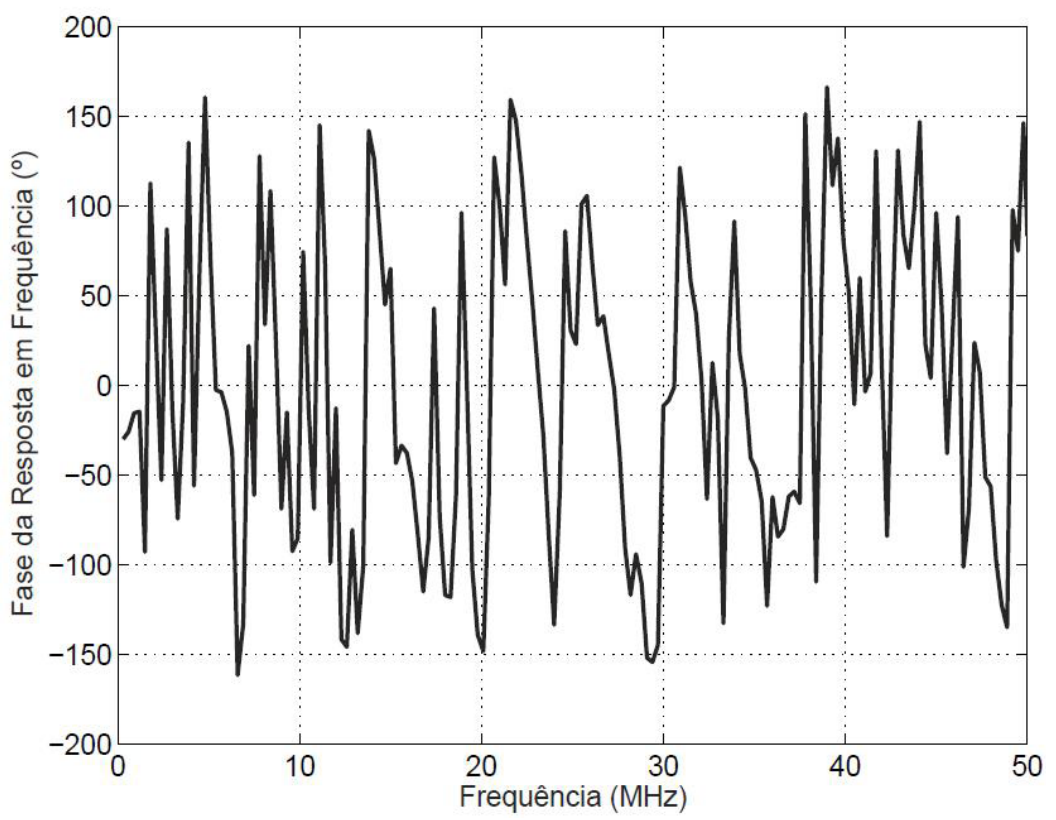

Figura 6. Fase da Resposta em frequência medida em um canal PLC. 
Ao analisar a figura 5, se observa que o comportamento da fase do canal em análise sofre variações frequentes e relacionadas às características dos circuitos (Vital, 2010).

A partir dos valores medidos em um processo de extração de parâmetros, pode-se realizar uma análise estatística dos efeitos de propagação de pequena escala de um canal e, consequentemente, determinar os parâmetros importantes no projeto de soluções de comunicações como função de autocorrelação, atrasos (médio e RMS) e banda de coerência. A partir das características do canal é possível definir os requisitos de projeto dos transceptores (conjunto de receptores e transmissores) como: taxa de transmissão (baud rate), potência de transmissão, sensibilidade do receptor e taxa de erro de bits (BER).

\section{Características das fontes ruidosas}

O ruído presente no canal PLC é o somatório dos cinco sinais interferentes apresentados na figura 3.

O ruído colorido é gerado por computadores, controladores de luminosidade de lâmpadas (dimmer's) e secadores de cabelo e, apresenta redução da densidade espectral de potência proporcional a um aumento da frequência.

O ruído de faixa-estreita é uma interferência provocada por serviços comerciais de rádio e aparelhos de televisão.

Retificadores e tiristores geram o ruído impulsivo periódico síncrono à rede de distribuição de energia elétrica com taxas de repetição múltiplas de $60 \mathrm{~Hz}$, caso do Brasil.

Na faixa entre 50 e $200 \mathrm{kHz}$ observa-se a presença do ruído impulsivo periódico assíncrono, que é gerado, principalmente, por circuitos que possuem fontes chaveadas.

O último tipo de ruído cujos efeitos podem ser indesejados na comunicação e que usa a Tecnologia PLC é o impulsivo assíncrono, visto que possui amplitudes superiores ao do ruído colorido e, ainda, com duração que pode gerar erros em rajada (Hrasnica, Haidine e Lehnert, 2004). A principal fonte desse tipo de ruído é a operação de diodos retificadores controlados (SCRs).

A figura 6 mostra o comportamento ruidoso no domínio da frequência medido em um laboratório e que segue os procedimentos utilizados por Vital (2010). 


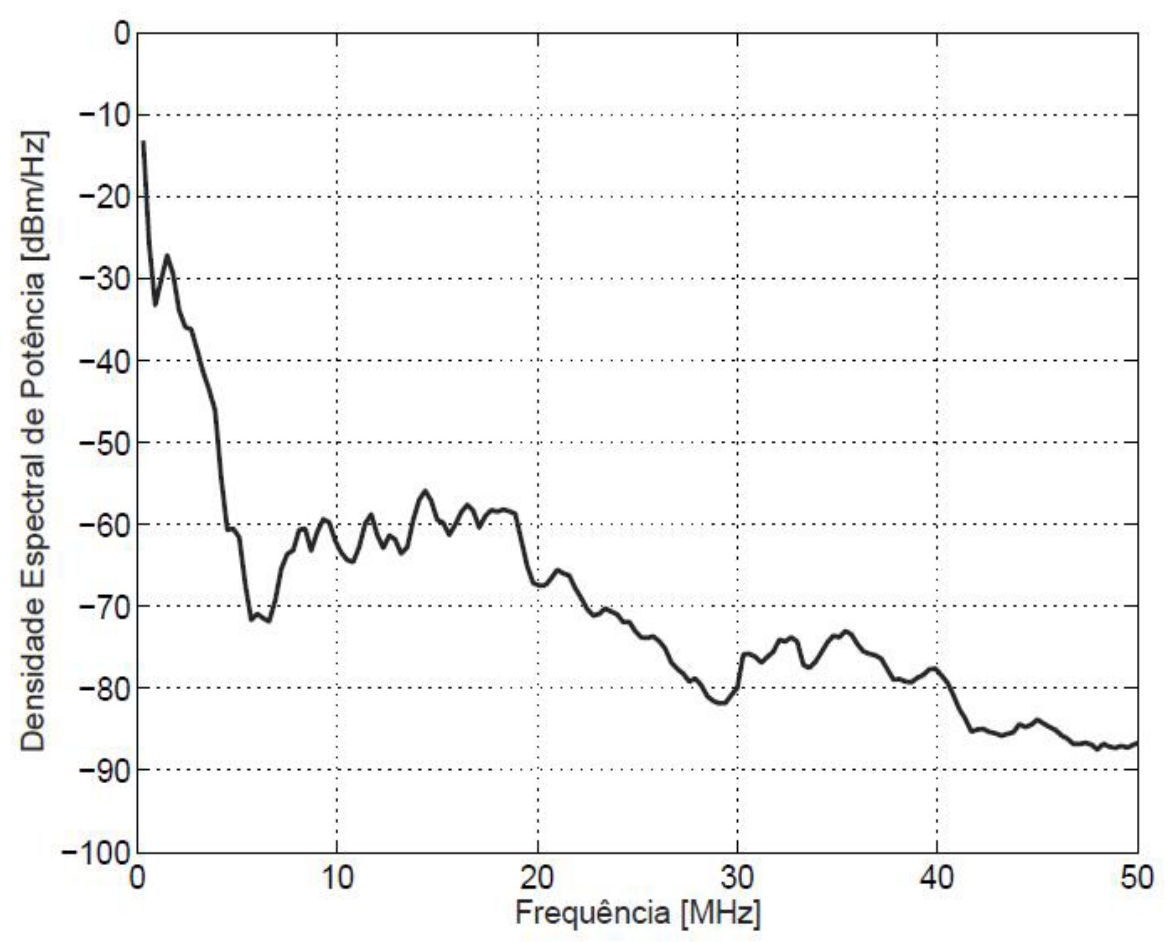

Figura 7. Densidade Espectral de Potência medida em um canal PLC

Ao analisar a figura 6 se observa forte influência do ruído colorido, uma vez que existe a redução da densidade espectral de potência frente ao aumento da frequência. Adicionalmente, pode-se destacar a existência de ruído de faixa-estreita entre 10 e 40 MHz.

\section{Conclusão}

Ao se analisarem as características da comunicação PLC oferecidas neste trabalho e os resultados apresentados por Vidal (2005) e Martins (2006), observa-se a aplicabilidade dessa tecnologia para o aumento de soluções que permitam comunicação de dados a altas taxas e preços competitivos. Para aumentar a difusão da tecnologia PLC no Brasil é necessário que novos investimentos em pesquisa e desenvolvimento sejam realizados, a fim de adequarem as soluções comerciais disponíveis, ou em projeto, às características das linhas de transmissão.

Dentreostemas de pesquisa emevidência nacomunidade científicamundial, a caracterização do canal de comunicação, assim como o comportamento ruidoso, tem papel importante na definição das características dos serviços comercializados e, consequentemente, no desenvolvimento de produtos eficientes. Portanto, torna-se necessário realizar avaliações de diferentes canais com maior variabilidade possível para garantir a operacionalidade de novos sistemas em qualquer ambiente brasileiro com qualidade satisfatória. 


\section{Referências}

Götz, M., Rapp, M., Dostert, K. (2004). "Power Line Channel Characteristics and Their Effect on Communication System Design", IEEE Communications Magazine, v. 42, pp. 78-96.

APTEL (2003). "Power Line Communications - PLC".

HomePlug Powerline Alliance (2006). "HomePlug Powerline Alliance Fact Sheet”.

Open PLC European Research Alliance (2005). "Theoretical Postulation of PLC Channel Model".

Philipps, H. (1999). "Modelling of Powerline Communications Channels", International Symposium on Power Line Communications, pp. 14-21.

Zimmermann, M., Dostert, K. (2002). “A Multipath Model for the Powerline Channel”, IEEE Transactions on Communications, v. 50, pp.553-559.

Hrasnica, H., Haidine, A., Lehnert, R. (2004). "Broadband Powerline Communications Network Design”, John Wiley and Sons, Londres.

Vital,R.B. N.(2010). “Caracterização do Canalde Comunicação em Redes de Distribuição de Energia Elétrica de Baixa Tensão”, Instituto Nacional de Telecomunicações, Santa Rita do Sapucaí.

Vidal, A. M. (2005). "Estudo do Estado da Arte e Análise de Desempenho de Sistemas de Comunicação PLC de Banda Larga", Universidade Federal de Santa Catarina, Florianópolis.

Martins, F. D. (2006). "Proposta de Arquitetura e Modelo de Negócios em Sistemas PLC”, Universidade Federal do Rio Grande do Sul, Porto Alegre. 